\section{VGLUT3, un acteur insoupçonné de la transmission cholinergique striatale}

Bénédicte Amilhon, Salah દl Mestikawy
Inserm U513, Neurobiologie et Psychiatrie, Université Pierre et Marie Curie,

Bâtiment B, $4^{e}$ étage, case courrier 37,

9, quai Saint Bernard, 75252 Paris Cedex 5, France.

Benedicte.Amilhon@snv.jussieu.fr

\section{Le glutamate et les VGLUT}

Le glutamate est non seulement un acide aminé essentiel au métabolisme de toute cellule, mais aussi le principal neurotransmetteur excitateur du cerveau (utilisé par plus de $30 \%$ des neurones). Les neurones glutamatergiques sont présents dans toutes les structures et participent à l'ensemble des fonctions cérébrales. Avant d'être libéré à l'arrivée d'un potentiel d'action, le glutamate, comme tous les neurotransmetteurs, est accumulé par des transporteurs dans des organites appelés vésicules synaptiques. Trois transporteurs vésiculaires du glutamate (VGLUTl à - 3) ont été découverts au début de ce siècle [1-3]. Ces protéines sont à la fois des marqueurs fonctionnels et anatomiques des neurones glutamatergiques. Depuis leur découverte, notre vision de la neurotransmission glutamatergique a considérablement évolué. Les trois VGLUT mettent en effet en lumière trois sous-systèmes glutama- tergiques, définis par la présence d'un des trois transporteurs vésiculaires. VGLUTl est présent dans les neurones du télencéphale, et assure $80 \%$ de la neurotransmission glutamatergique $[4$, 5]. VGLUT2 est principalement localisé dans des neurones du diencéphale, avec une distribution complémentaire à celle de VGLUTl $[6,7]$. Les neurones exprimant VGLUT1 et VGLUT2 couvrent à eux deux l'ensemble des neurones «classiquement » définis comme glutamatergiques.

L'étude de souris mutantes n'exprimant plus l'un de ces deux transporteurs montre que l'invalidation de VGLUTI ou de VGLUT2 a des conséquences très différentes, mais qu'ils sont tous deux vitaux pour le fonctionnement du cerveau [5, 8]. Ces travaux ont aussi établi de façon indiscutable que la capacité à libérer du glutamate et la taille du quantum dépendent directement de la présence et du nombre de VGLUT par vésicule $[5,9]$.
A

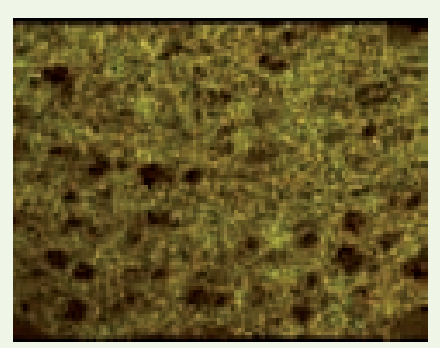

B

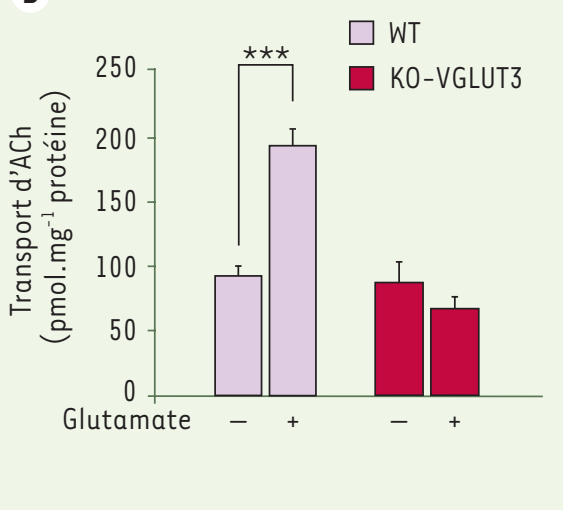

\section{L'originalité de VGLUT3}

VGLUT3 se démarque nettement des deux autres isoformes. Son expression chez l'adulte est beaucoup plus discrète et surprenante. En effet, elle est restreinte à des sous-populations neuronales décrites comme non glutamatergiques. On le trouve notamment dans certains interneurones GABAergiques du cortex et de l'hippocampe, dans les neurones sérotoninergiques du raphé et enfin dans les interneurones cholinergiques du striatum, auxquels nous nous sommes plus particulièrement intéressés [3, 10]. Cette présence insolite (Figure 1) pose la question d'une possible cotransmission (colibération de deux neurotransmetteurs habituellement non associés).

Le striatum fait partie d'un ensemble de structures cérébrales appelées les ganglions de la base. II participe à des fonctions variées et complexes allant des fonctions motrices aux fonctions cognitives [11]. L'activité des neurones

Figure 1. Coexpression de VGLUT3 et VAChT. A. Colocalisation en immunofluorescence de VGLUT3 et VAChT dans le striatum de souris sauvages. VGLUT3 est détecté en rouge et VAChT en vert, la superposition des deux marquages montre un colocalisation quasi parfaite des deux transporteurs vésiculaires. B. Transport d'ACh- ${ }^{3} \mathrm{H}$ sur une préparation de vésicules synaptiques striatales de souris sauvages (WT) ou KO-VGLUT3. Dans le cas de vésicules striatales de souris sauvages, les vésicules mises en présence d'ACh et de glutamate transportent plus d'ACh. Cet effet du glutamate sur le transport d'ACh disparaît chez les souris KO-VGLUT3. 
de projection du striatum est sous le contrôle des afférences glutamatergiques du cortex et du thalamus. Localement, ces neurones de projection reçoivent une double innervation cholinergique (des interneurones) et dopaminergique (de la voie nigro-striée). L'acétylcholine (ACh) et la dopamine (DA) agissent de façon opposée et complémentaire, créant une balance ACh/DA dans le striatum [12].

La génération de souris invalidées pour le gène de VGLUT3 (souris KO-VGLUT3) nous a permis de faire un pas en avant dans la compréhension du rôle de ce transporteur vésiculaire atypique. VGLUT3 est particulièrement abondant dans le striatum où il est exprimé par tous les interneurones cholinergiques et sa localisation se superpose presque parfaitement à celle du transporteur vésiculaire de l'acétycholine (VAChT, Figure IA). Notre choix s'est porté dans un premier temps sur l'étude de la fonction de VGLUT3 dans ces importants interneurones modulateurs/intégrateurs striataux [13].

\section{La perte de VGLUT3}

entraîne une diminution

\section{de la transmission cholinergique}

Nous avons d'abord analysé les capacités motrices de nos souris KO-VGLUT3. L'injection d'un neuroleptique, l'halopéridol, se traduit par une certaine raideur des membres et une difficulté de l'animal à initier un mouvement volontaire (état aussi appelé catalepsie). Nous avons observé que les souris n'exprimant plus VGLUT3 sont moins sensibles à l'effet de l'halopéridol que des souris sauvages. De plus, elles ont une activité locomotrice spontanée plus élevée que celle des souris contrôles, ainsi qu'une hypersensiblité à l'effet hyperlocomoteur de la cocaïne. Un phénotype identique avait été préalablement observé dans un modèle d'hypocholinergie striatale obtenu par l'ablation sélective des interneurones cholinergiques [14]. Mais l'hypothèse d'une modification de la neurotransmission dopaminergique dans le striatum permettrait également d'expliquer ces différences comportementales.

Pour trancher entre ces deux possibilités, nous avons testé l'effet de l'injection d'un inhibiteur de l'enzyme de dégradation de l'ACh agissant au niveau du système nerveux central. Le traitement par le donépezil permet de rétablir chez les animaux KO-VGLUT3 une activité locomotrice semblable à celles des souris sauvages. La «normalisation » des taux extra-synaptiques d'ACh permet donc de corriger le phénotype hyperlocomoteur des souris KO-VGLUT3.

La validité de cette hypothèse hypocholinergique a été ensuite directement mise à l'épreuve en mesurant la capacité de libération d'ACh tritiée $\left(\mathrm{ACh}-{ }^{3} \mathrm{H}\right)$ in vitro sur des tranches de striatum, chez les souris sauvages et mutantes. Le striatum issu des animaux KO-VGLUT3 libère moins d'ACh, alors que dans les mêmes conditions expérimentales, la libération de DA est équivalente entre les deux génotypes.

\section{Un nouveau mécanisme \\ de régulation présynaptique : \\ la «synergie vésiculaire 》}

La perte de VGLUT3 a donc comme conséquence d'amoindrir le tonus cholinergique striatal. Mais quel mécanisme moléculaire est-il à l'origine de cet effet? Nous avons choisi d'explorer l'hypothèse d'une influence directe de la capture vésiculaire de glutamate sur le quantum vésiculaire d'ACh. Dans un premier temps, nous avons montré par immunoprécipitation que VGLUT3 et VAChT sont présents sur les mêmes vésicules dans les terminaisons nerveuses des interneurones cholinergiques.

II semble donc que glutamate et ACh peuvent être accumulés au sein des mêmes vésicules. L'effet de ce cotransport a été mesuré sur des préparations de vésicules striatales mises en présence d'ACh- ${ }^{3} \mathrm{H}$. La quantité d'ACh $-{ }^{3} \mathrm{H}$ transportée au cours du temps atteint une valeur supérieure de plateau lorsque du glutamate est présent dans le milieu de transport. Cet effet du glutamate existe uniquement dans les régions du cerveau où VGLUT3 et VAChT

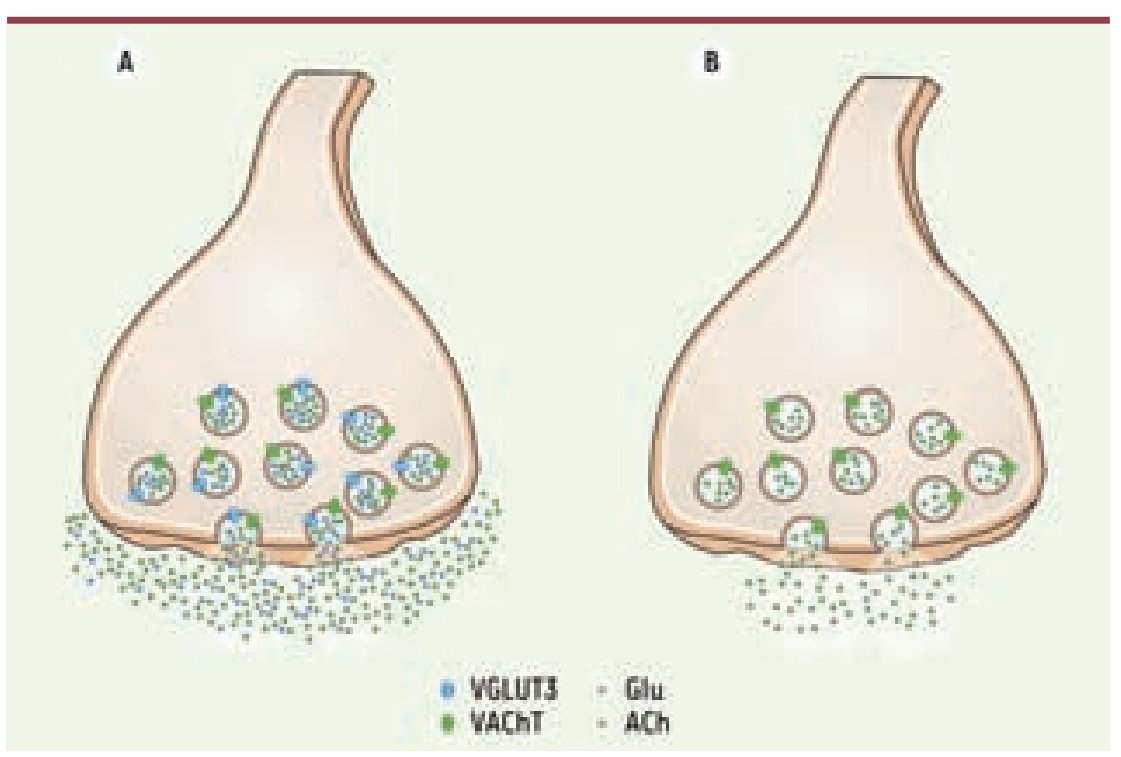

Figure 2. Mécanisme de la «synergie vésiculaire ». A. Représentation schématique d'une terminaison cholinergique striatale chez une souris sauvage. La présence de VGLUT3 sur les mêmes vésicules que VAChT permet une accumulation plus importante d'ACh dans chaque vésicule. La quantité d'ACh libérée est donc augmentée elle aussi. Ce mécanisme est appellé synergie vésiculaire. B. Terminaison cholinergique striatale chez une souris $\mathrm{KO}$ VGLUT3. La perte de VGLUT3 dans ces terminaisons provoque une diminution de l'accumulation et de la libération d'ACh. 
sont colocalisés, et disparaît en présence d'inhibiteurs de VGLUT3. Mais surtout, cet effet disparaît sur des préparations de vésicules de striatum de souris KOVGLUT3 (Figure IB).

Le glutamate (via VGLUT3) potentialise donc l'accumulation vésiculaire d'ACh. Nous avons nommé ce mécanisme de régulation présynaptique «synergie vésiculaire » (Figure 2).

Des rôles nouveaux pour le glutamate et VGLUT3 Notre travail montre donc que les rôles de cet acide aminé multifonctionnel qu'est le glutamate sont encore plus étendus que ce qui était soupçonné jusqu'à présent. Via son transport dans les vésicules synaptiques, le glutamate permet une modulation intra-synaptique de la neurotransmission cholinergique. Cette fonction inattendue du glutamate ouvre de nombreuses perspectives de recherche.
II reste à explorer les fonctions du glutamate colibéré avec I'ACh, ou encore la possibilité de moduler pharmacologiquement la transmission cholinergique striatale, en ciblant VGLUT3 au cours du développement ou dans la maladie de Parkinson. $\diamond$

VGLUT3 and the mechanism of vesicular synergy

\section{RÉFÉRENCES}

1. Bellocchio દદ, Reimer RJ, Fremeau RT, Edwards RH. Uptake of glutamate into synaptic vesicles by an inorganic phosphate transporter. Science 2000 ; 289: 957-60.

2. Herzog $\varepsilon$, Bellenchi GC, Gras C, et al. The existence of a second vesicular glutamate transporter specifies subpopulations of glutamatergic neurons. J Neurosci $2001 ; 21:$ RC181.

3. Gras C, Herzog $\varepsilon$, Bellenchi GC, et al. A third vesicular glutamate transporter expressed by cholinergic and serotoninergic neurons. J Neurosci $2002 ; 22$ : 5442-51.

4. Fremeau RT Jr, Kam K, Qureshi T, et al. Vesicular glutamate transporters 1 and 2 target to functionally distinct synaptic release sites. Science 1994; 304 : 1815-9.
5. Wojcik SM, Rhee JS, Herzog $\varepsilon$, et al. An essential role for vesicular glutamate transporter l (VGLUTI) in postnatal development and control of quantal size. Proc Natl Acad Sci USA 2004 ; 101 : 7158-63.

6. Gras C, Vinatier J, Amilhon B, et al. Developmentally regulated expression of VGLUT3 during early postnatal life. Neuropharmacology 2005 ; 49 : 901-11.

7. Herzog $\varepsilon$. Understanding glutamatergic neurons in mammalians: «ménage à trois 》. Med Sci (Paris) 2000 ; $20: 1063-6$.

8. Moechars D, Weston MC, Leo S, et al. Vesicular glutamate transporter VGLUT2 expression levels control quantal size and neuropathic pain. J Neurosci $2006 ; 26: 12055-66$

9. Takamori S. VGLUTs: exciting times for glutamatergic research? J Neurosci 2006 ; 55 : 343-51.

10. Herzog $\varepsilon$, Gilchrist J, Gras C, et al. Localization of VGLUT3, the vesicular glutamate transporter type 3 , in the rat brain. Neuroscience $2004 ; 123: 983-1002$

11. Voorn P, Vanderschuren LJ, Groenewegen HJ, et al. Putting a spin on the dorsal-ventral divide of the striatum. Trends Neurosci 2004 ; 27 : 468-474

12. Calabresi P, Centonze $D$, Gubellini $P$, et al. Acetylcholine-mediated modulation of striatal function. Trends Neurosci $2000 ; 23: 120-6$.

13. Gras C, Amilhon B, Lepicard EM, et al. The vesicular glutamate transporter VGLUT3 synergizes striatal acetylcholine tone. Nat Neurosci 2008 ; 11 : 292-300.

14. Kitabatake Y, Hikida T, Watanabe D, Pastan I, Nakanishi S. Impairment of reward-related learning by cholinergic cell ablation in the striatum. Proc Natl Acad Sci USA 2003 ; 100 : 7965-70.

\section{Un ouvrage unique qui traite d'un champ très vaste de la pathologie}

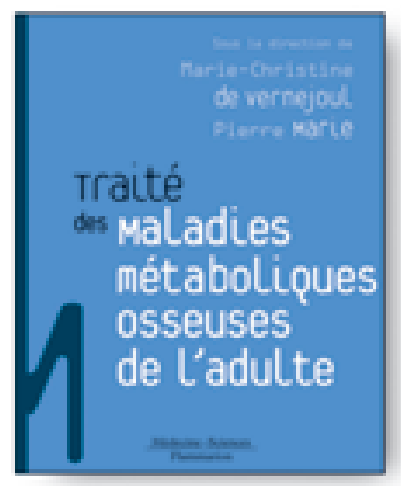

26 noveribe 2006 - 368 agcrs

250 Illustrations en couleurs

Phis puble $T$ TC : 956

\section{Maladies métaboliques osseuses de I'adulte}

Marie-Christine DE VERNEJOUL, Pierre MARIE

- Les affections étudićes relkvent à la fois de la rhumatologie, de l'endocrinologie, de l'orthopédie et de la cancérologie.

- Après Tétude de la physiologie osseuse à Pàge adulte et de la régulation du métabolisme osseux, les autcurs exposent les moyens d'tétede de la qualité osseuse et d'évaluation de la fragilité de fos.

- Puis, ils abordent les différentes pathologies osscuses, avec un chapitre important sar Tostéoporose: definitios, épidémiologie, génteique, ostéoporose de la femme, de l'homme, ostéoporose de la corticothérapie, tous ses traitemenes et ses stratćgies thítapeutiques les mieux adapetes.

- La dernière partic expose toutes les aetres ostéopathies : les métastases osscuses, les affections parathyroidiennes, les ostéomalacies, la maladie de Paget, ete.

\section{Au total, un ouvrage de référence et très richement illustré, indispensable à la pratique quotidienne de nombreuses spécialités.}

En vente chez votre libraire specialise, par correspondance ou sur notre site wwwmedecine.flammarion.com

8

Bon de commande à retourner complèté a : FLAMMARION Médecine-Sciences - 87, quai Panhard et Levassor - 75647 Paris cedex 13

Maladies metaboliques osseuses de Fadulte : 95 C TTC Quantité (France metropolitaine uniquement. Autres nous consulter)

( +5 e de participation aux frais de port) soit $100 \mathrm{C}$ Je joins mon réglement a la commande : Montamt total de :

Cheque bancaire ou postal payable en France a Prordre de Flammarion SA (Une facture acquittee sera jointe au colin)

Carte bancaire $n^{*}$

Date drexpiration :

\begin{tabular}{|l|l|l|}
\hline & 1 & 1 \\
\hline
\end{tabular}

Les 3 derniers chiffres situts au dos de votre carte bancaire :

Fonction $/$ sptcialite : .......................... Adresse :

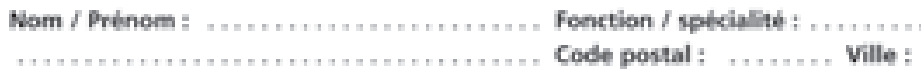

Tel: : E-mail :

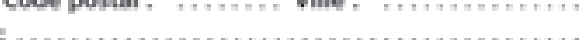

Date et signature obligatoire:

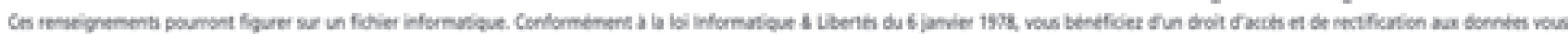
concernant 